\title{
Performance of Arrhenotokous and Thelytokous Thrips tabaci (Thysanoptera: Thripidae) on Onion and Cabbage and Its Implications on Evolution and Pest Management
}

\author{
XIAO-WEI LI, ${ }^{1,2}$ JOZSEF FAIL, ${ }^{3}$ PING WANG, ${ }^{1}$ JI-NIAN FENG ${ }^{2}$ and A. M. SHELTON ${ }^{1,4}$
}

J. Econ. Entomol. 107(4): 1526-1534 (2014); DOI: http://dx.doi.org/10.1603/EC14070

\begin{abstract}
Onion thrips, Thrips tabaci Lindeman (Thysanoptera: Thripidae), is an important pest on onion and cabbage. Two reproductive modes - arrhenotoky and thelytoky - are found in this species and co-occur in the field. We compared life table traits between arrhenotokous and thelytokous T. tabaci on cabbage and onion. Experiments were conducted in cages to determine which reproductive mode is more competitive. Additionally, host adaption of the arrhenotokous and thelytokous T. tabaci between onion and cabbage was investigated. On onion, arrhenotokous T. tabaci performed better than thelytokous T. tabaci, while on cabbage the opposite occurred. When comparing life table and demographic growth parameters (net reproductive rates $R_{0}$, mean generation time $T$, the intrinsic rate of natural increase $r_{\mathrm{m}}$, finite rate of increase $\lambda$, and population doubling time $T_{\mathrm{d}}$ ) on different host plants, we found that arrhenotokous T. tabaci performed better on onion than on cabbage, whereas thelytokous T. tabaci performed better on cabbage than on onion. Host-related performance differences in this species suggest that the divergence between two reproductive modes might be associated with host adaption. Pest management strategies for this global pest should recognize that the two reproductive modes can impact population dynamics on different crops.
\end{abstract}

KEY WORDS onion thrips, onion, cabbage, life table trait

Onion thrips, Thrips tabaci Lindeman (Thysanoptera: Thripidae), is a global pest on many important agricultural crops including onion, leek, cabbage, tobacco, tomato, and cotton. T. tabaci has become an increasing concern in commercial onion (Allium cepa $\mathrm{L}$.) due to resistance to many insecticides, its transmission of plant viruses, and rapid development and high reproductive capacity (Diaz-Montano et al. 2011). In North America and many other parts of the world, T. tabaci is also a consistent and severe pest on cabbage, Brassica oleracea capitata (L.) (Wolfenbarger and Hibbs 1958; Shelton et al. 1998; Trdan et al. 2007, 2008).

There are two reproductive modes in $T$. tabaci: thelytoky (asexual reproduction) and arrhenotoky (sexual reproduction; Jenser and Szénási 2004). In thelytokous populations, only females exist and they are parthenogenetically developed from unfertilized eggs. Arrhenotokous populations consist of both males and females: diploid females are produced from fertilized eggs and haploid males from unfertilized eggs (Moritz 1997).

\footnotetext{
${ }^{1}$ Department of Entomology, Cornell University, New York State Agricultural Experiment Station, Geneva, NY 14456.

${ }^{2}$ Key Laboratory of Plant Protection Resources and Pest Management, Ministry of Education, Northwest A\&F University, Yangling, Shaanxi 712100, China.

${ }^{3}$ Department of Entomology, Faculty of Horticultural Science, Corvinus University of Budapest, Budapest, 1118, Hungary.

${ }^{4}$ Corresponding author, e-mail: ams5@cornell.edu.
}

These two reproductive modes may coexist in populations collected in the field (Nault et al. 2006), but it is difficult to distinguish between thelytokous and arrhenotokous female adults morphologically (Jenser and Szénási 2004). However, the two reproductive modes differ ecologically and genetically, including having different host preferences. Arrhenotokous $T$. tabaci collected from tobacco proliferated well on both tobacco and onion, whereas both arrhenotokous and thelytokous populations from leek could not survive on tobacco (Chatzivassiliou et al. 2002). Strong host specificities of T. tabaci have been reported for populations in North America and the Southern Hemisphere (Sakimura 1962).

Based on host adaption, reproductive modes, and mitochondrial DNA sequences, T. tabaci are divided into two types: the arrhenotokous tobacco type, which is found on tobacco (Zawirska 1976, Brunner et al. 2004, Toda and Murai 2007), and the leek type, which is polyphagous and has both reproduction modesarrhenotoky and thelytoky (Brunner et al. 2004, Toda and Murai 2007, Kobayashi and Hasegawa 2012). The two reproductive modes of the leek type can be discriminated using reproductive mode-specific primers based on sequence differences in the mitochondrial cytochrome c oxidase subunit I (COI) gene between the arrhenotokous and thyletokous strains (Kobayashi and Hasegawa 2012). 
The parthenogenetic and sexual populations of $T$. tabaci differ in their ability to transmit plant viruses. Arrhenotokous populations collected from tobacco were highly effective in transmitting tomato spotted wilt virus (TSWV; Chatzivassiliou et al. 2002), whereas arrhenotokous populations from leek transmit TSWV inefficiently (Wijkamp et al. 1995, Chatzivassiliou et al. 1999, 2002). By contrast, thelytokous populations did not transmit (Wijkamp et al. 1995, Chatzivassiliou et al. 2002) or transmitted TSWV poorly (Tedeschi et al. 2001), although recent studies reported that some thelytokous specimens were fairly efficient vectors (Jacobson and Kennedy 2013, Westmore et al. 2013).

Although there is strong host adaption for tobacco and nontobacco host plants in different strains of $T$. tabaci (Chatzivassiliou et al. 2002, Fekrat et al. 2009), there are no comparative life table data for polyphagous arrhenotokous and thelytokous populations and their host adaption. T. tabaci of the two reproductive modes were found to coexist in the field, and the frequency of these two reproductive modes varied temporally (Nault et al. 2006). Comparative life table data of arrhenotokous and thelytokous T. tabaci can help explain their biological differences and provide important information about their population dynamics in the field.

In our study, we examined the development and reproduction of arrhenotokous and thelytokous $T$. tabaci on two important host plants, onion and cabbage. Life table and demographic growth parameters were compared between the two reproductive modes, and competition experiments were conducted to determine which reproductive mode is more competitive. In addition, host adaption of arrhenotokous and thelytokous T. tabaci on two different host plants was investigated by comparing the life table and demographic growth parameters.

\section{Materials and Methods}

Population Maintenance and Insect Rearing. The arrhenotokous and thelytokous populations used in this study were established in 2011 from adult T. tabaci collected from cabbage in a research field (GPS coordinates: 42.873621, - 77.029556) of Cornell University, New York State Agricultural Experiment Station, Geneva, NY. Populations were maintained on potted onion plants or cabbage heads in environmental growth chambers at $20 \pm 1^{\circ} \mathrm{C}, 60 \pm 5 \%$ relative humidity (RH), and a photoperiod of 16:8 (L:D) h. To test whether the source host plants had effects on $T$. tabaci development and reproduction, arrhenotokous and thelytokous T. tabaci on onion were collected from onion fields in Peru, NY, and onion fields in Penn Yan, NY, respectively.

Host Plants. Two host plants, onion and cabbage, were used in this study. Onion sets and cabbage seeds were planted in plastic pots in a thrips-free growing chamber under conditions of $25 \pm 2^{\circ} \mathrm{C}, 70 \pm 5 \% \mathrm{RH}$, and a photoperiod of 16:8 (L:D) h. Onion plants $3 \mathrm{wk}$ old and cabbage plants 2 mo old were used in both life table studies and cage experiments.
Treatments. Four treatments were established to test arrhenotokous and thelytokous T. tabaci life table parameters and host adaption using lab-maintained colonies (T. tabaci collected from cabbage): 1) arrhenotokous T. tabaci on onion, 2) arrhenotokous $T$. tabaci on cabbage, 3 ) thelytokous T. tabaci on onion, and 4) thelytokous T. tabaci on cabbage.

To test whether source host plants had effects on development and reproduction of T. tabaci in their two reproductive modes, two additional treatments were added: 5) arrhenotokous T. tabaci collected from onion on onion and 6) thelytokous T. tabaci collected from onion on onion. Fitness and demographic growth parameters were compared between treatments 1) and 5) and between treatments 3) and 6).

Life Table Studies. For life table experiments, $T$. tabaci in each treatment were incubated individually in 1.7-ml microcentrifuge tubes and held at $20 \pm 1^{\circ} \mathrm{C}$, $60 \pm 5 \% \mathrm{RH}$, and a photoperiod of 16:8 (L:D) h. To start experiments, female adults were collected from arrhenotokous and thelytokous populations and left in tubes with a single piece of onion or cabbage leaf tissue ( 6 by $6 \mathrm{~mm}$ ) for oviposition. The number of eggs in the leaves was counted using the bottom light of a stereomicroscope (ZEISS, Stemi 2000, Carl Zeiss Microscopy, Jena, Germany). Leaf tissues with eggs were checked every $12 \mathrm{~h}$ for newly emerged larvae. First instars were transferred individually to new tubes with fresh leaf tissue. Egg durations and hatching rates were recorded. Thrips in immature stages were checked every $12 \mathrm{~h}$ for development, but the sex could not be determined until adulthood. Survivorship and developmental times at different stages were recorded. When adults emerged, arrhenotokous and thelytokous females were contained individually in tubes with leaf tissues. Arrhenotokous females were paired with males for $2 \mathrm{~d}$. Leaf tissues were changed at 24-h intervals and the number of eggs produced by each female was recorded. For arrhenotokous females, leaf tissues with eggs were moved into new tubes for analysis of daily sex ratios when the eggs developed into adults. Longevity, lifetime fecundity, and mean daily fecundity (lifetime fecundity divided by longevity) were calculated for each female.

Demographic Growth Parameters. Immature duration (egg laying to adult emergence), survival rate, daily fecundity, and daily sex ratio of offspring (in arrhenotokous treatments) were used to construct $l_{\chi} m_{\chi}$ life tables $\left(l_{\chi} m_{\chi}\right.$ is the net female maternity, $l_{\chi}$ is the percentage of females alive at age $\chi$, and $m_{\chi}$ is the mean number of female offspring produced by a female at age $\chi$ ) from which the following demographic growth parameters were calculated: net reproductive rates $\left(R_{0}=\Sigma l_{\chi} m_{\chi}\right)$, mean generation time $(T=$ $\left.\Sigma \chi l_{\chi} m_{\chi} / R_{0}\right)$, the intrinsic rate of natural increase $\left(r_{\mathrm{m}}=\ln R_{0} / T\right)$, finite rate of increase $\left[\lambda=\exp \left(r_{\mathrm{m}}\right)\right]$, and population doubling time $\left[T_{\mathrm{d}}=\ln (2) / r_{\mathrm{m}}\right]$ (Carey 1993).

Mean demographic parameter estimates with standard errors were generated using the jackknife technique (Meyer et al. 1986, Maia et al. 2000). To apply the jackknife technique, we first calculated the pop- 
ulation parameters based on all $(n)$ individuals in each treatment. For example, we calculated the intrinsic rate $r_{\text {all }}$ based on all individuals. We then omitted individual $\mathrm{i}$ and used the other $n-1$ individuals to calculate the jackknife value of $r_{\text {i-jack }}$. Next, we calculated pseudovalue $r_{\text {i-pseudo }}$ as $r_{\text {i-pseudo }}=n \times r_{\text {all }}-$ $(n-1) \times r_{\text {i-jack }}$. We repeated this process until pseudovalues were calculated for all $n$ possible omissions from the original data set. Then we used $n$ pseudovalue $r_{\text {i-pseudo }}$ to calculate means and standard errors. The same method was used for $R_{0}, T, \lambda$, and $T_{\mathrm{d}}$.

Competition Experiments in Cages Between Arrhenotokous and Thelytokous T. tabaci. Cage experiments were set up to observe changes in the relative ratios of two reproductive modes in mixed populations on onion and cabbage. Experiments were conducted in thrips-proof insect rearing cages (BugDorm- $44545 \mathrm{~F}$ Insect Rearing Cage, 47.5 by 47.5 by $47.5 \mathrm{~cm}$ with 150 mesh Nylon) with three cages (replications) per treatment. On each host plant, three treatments were set up: arrhenotokous T. tabaci single population; arrhenotokous and thelytokous T. tabaci mixed population; thelytokous T. tabaci single population. Cages were kept in environmental growth chambers at $20 \pm 1^{\circ} \mathrm{C}$, $60 \pm 5 \% \mathrm{RH}$, and a photoperiod of 16:8 (L:D) h, the same conditions as life table experiments. To start the mixed cages, 10 arrhenotokous (mated, paired with males for $2 \mathrm{~d}$ ) and 10 thelytokous 3- to 5-d-old females were released into each cage. For the control single cages, 20 arrhenotokous (mated, paired with males for $2 \mathrm{~d}$ ) or thelytokous 3- to 5-d-old females were released into each cage. Each cage contained five pots of green onion plants at the 6-8 leaf stage or two pots of cabbage plants at the 10-12 leaf stage.

Sampling started one month after placing the insects in the cages; two of five pots of onions in the onion cages or one of two pots of cabbage in the cabbage cages were replaced with new plants. All the adults on removed plants were collected and preserved in $70 \%$ ethanol. Sampling was conducted every $20 \mathrm{~d}$ for five times. Twenty adults were randomly selected from each sample and were examined by PCR (polymerase chain reaction) analysis for molecular identification of their reproductive mode using reproductive mode-specific $C O I$ gene primers. The percentage of thelytokous T. tabaci in each sample was calculated.

Identification of Reproductive Mode by PCR Analysis. To verify the reproductive mode-specific single nucleotide polymorphisms (SNPs) in the COI gene identified by Kobayashi and Hasegawa (2012) in the $T$. tabaci colonies used in this study, the COI fragment was amplified by PCR from five individuals from the arrhenotokous colony and five individuals from the thelytokous colony and sequenced. DNA from individual thrips was prepared using a rapid DNA extraction method (Tiewsiri and Wang 2011). A single thrips was homogenized in $20 \mu \mathrm{l}$ tissue lysis buffer $(10 \mathrm{mM}$ Tris-HCl, pH 8.3, $50 \mathrm{mM} \mathrm{KCl,} 2.5 \mathrm{mM} \mathrm{MgCl}, 0.45 \%$ Nonidet P-40, $0.45 \%$ Tween $20,0.01 \%$ gelatin, and 60 $\mu \mathrm{g}$ proteinase $\mathrm{K}$ ) and incubated at $65^{\circ} \mathrm{C}$ for $30 \mathrm{~min}$, followed by an incubation at $95^{\circ} \mathrm{C}$ for $15 \mathrm{~min}$ to inac- tivate the proteinase $\mathrm{K}$. The lysate was used directly for PCR amplification of the COI fragment, using the universal COI primer pair, LepF: $5^{\prime}$-ATTCAACCAAT CATAAAGATATTGG-3' and LepR: 5'-TAAACTTC TGGATGTCCAAAAAATCA-3' (Hebert et al. 2004). The PCR product was processed using a one-step enzymatic purification procedure (Dorit et al. 2001) and subject to DNA sequencing.

PCR analysis to differentiate the arrhenotokous and thelytokous T. tabaci was designed based on the reproductive mode-specific SNPs in the COI gene in $T$. tabaci (Kobayashi and Hasegawa 2012), and confirmed by our DNA sequencing of the COI fragment from five arrhenotokous and five thelytokous T. tabaci from our lab colonies. A reproductive mode diagnostic PCR analysis was established to include in the PCR reaction three primers, the arrhenotokous-specific primer (5'-AACAGCTATTCTCCTTCTTTTATCTC$\left.3^{\prime}\right)$ and thelytokous-specific primer (5'-GAACAGTA TATCCACCTTTATCAACG-3'; Kobayashi and Hasegawa 2012) and a generic primer (5'-TAAACTTCT GGGTGACCAAAAAATCA-3'). A PCR product of 161 bp is diagnostic for the arrhenotokous strain and 351 bp for thelytokous strain.

Statistical Analysis. All data analyses were performed in SPSS (SPSS Inc., 2007 Chicago, IL,). Before analysis, data were checked for normality using nonparametric Kolmogorov-Smirnov test $(P<0.05)$ and all percentage data were arcsine or square root transformed, as necessary, but untransformed means are presented. For the data that were not normal (developmental times and survival rates at each immature stage between two host plants in each reproductive mode and between two reproductive modes on each host plant), direct estimates were compared using the nonparametric Mann-Whitney $U$ test $(P<0.05)$. For the data that followed normal distribution (female adult longevity, total fecundity, daily fecundity, and five demographic growth parameters), a two-way ANOVA was used to analyze the effects of host plants, reproductive modes, and their interactions $(P<0.05)$. Pairwise comparisons among main effects were performed using LSD test for multiple comparisons $(P<0.05)$.

In cage experiments, percentage changes of thelytokous T. tabaci in samples on two host plants over time were compared using one-way repeated measures ANOVA.

\section{Results}

The Effects of Thrips Sources on Fitness and Demographic Growth Parameters of Arrhenotkous and Thelytokous $\boldsymbol{T}$. tabaci. Thrips sources had no effects on fitness and demographic growth parameters in arrhenotokous and thelytokous T. tabaci. There were no statistical differences in longevity, total fecundity, daily fecundity, and five demographic growth parameters between arrhenotokous T. tabaci collected from onion and cabbage (Table 1). The same result was also found in thelytokous T. tabaci (Table 1), so the data 
Table 1. Comparisons of fitness and demographic growth parameters [mean $\pm \mathrm{SE}(\boldsymbol{n})]$ of arrhenotokous and thelytokous $\mathrm{T}$. $t a b a c i$ collected from cabbage and onion when they were reared on onion

\begin{tabular}{|c|c|c|c|c|c|c|c|c|}
\hline \multirow{2}{*}{ Parameter } & \multicolumn{4}{|c|}{ Arrhenotokous T. tabaci } & \multicolumn{4}{|c|}{ Thelytokous T. tabaci } \\
\hline & From cabbage & From onion & $t$ & $P$ & From cabbage & From onion & $t$ & $P$ \\
\hline Longevity & $31.6 \pm 3.9(20)$ & $29.1 \pm 2.1(49)$ & 0.61 & 0.547 & $29.8 \pm 3.6(17)$ & $28.0 \pm 3.3(29)$ & 0.35 & 0.728 \\
\hline Total fecundity & $145.3 \pm 21.7(20)$ & $107.8 \pm 12.6(49)$ & 1.56 & 0.124 & $90.0 \pm 14.8(17)$ & $81.9 \pm 13.5(29)$ & 0.39 & 0.702 \\
\hline Daily fecundity & $3.9 \pm 0.4(20)$ & $3.2 \pm 0.3(49)$ & 1.31 & 0.194 & $2.7 \pm 0.3(17)$ & $2.3 \pm 0.3(29)$ & 0.81 & 0.421 \\
\hline$R_{0}$ & $56.7 \pm 8.6(20)$ & $49.2 \pm 6.0(49)$ & 0.70 & 0.487 & $17.8 \pm 2.9(17)$ & $21.9 \pm 3.6(29)$ & -0.77 & 0.444 \\
\hline$T$ & $41.0 \pm 1.4(20)$ & $41.3 \pm 1.1(49)$ & -0.12 & 0.909 & $40.5 \pm 1.9(17)$ & $43.8 \pm 2.1(29)$ & -1.04 & 0.302 \\
\hline$r_{\mathrm{m}}$ & $0.099 \pm 0.003(20)$ & $0.095 \pm 0.002(49)$ & 0.98 & 0.330 & $0.071 \pm 0.003(17)$ & $0.071 \pm 0.003(29)$ & 0.16 & 0.878 \\
\hline$\lambda$ & $1.104 \pm 0.003(20)$ & $1.100 \pm 0.003(49)$ & 0.98 & 0.330 & $1.074 \pm 0.003(17)$ & $1.073 \pm 0.036(29)$ & 0.16 & 0.878 \\
\hline$T_{\mathrm{d}}$ & $7.0 \pm 0.2(20)$ & $7.3 \pm 0.2(49)$ & -0.96 & 0.339 & $9.7 \pm 0.4(17)$ & $9.8 \pm 0.5(29)$ & -0.15 & 0.883 \\
\hline
\end{tabular}

Normal data. Means within the same rows in each reproductive mode were compared by Student's $t$-test at $P<0.05$ level.

$n$, number of replications in each treatment; $R_{0}$, net reproductive rates; $T$, mean generation time; $r_{\mathrm{m}}$, the intrinsic rate of natural increase; $\lambda$, finite rate of increase; $T_{\mathrm{d}}$, population doubling time.

were pooled from treatments 1) and 5) and data from treatments 3) and 6).

Comparison of Life Table Parameters Between Arrhenotokous and Thelytokous $T$. tabaci on Onion and Cabbage Plants. Mean Developmental Times of Immature Arrhenotokous and Thelytokous T. tabaci on Two Different Host Plants. On onion, except for the egg stage $(P=0.146)$, developmental times of first instar, second instar, prepupa, and pupa stages in thelytokous T. tabaci were significantly longer than those in arrhenotokous thrips $(P<0.0001$; Table 2$)$. Noticeably, the duration of the second instar in thelytokous $T$. tabaci was $\approx 1 \mathrm{~d}$ longer than in arrhenotokous thrips and the entire immature duration was $\approx 2 \mathrm{~d}$ longer in thelytokous T. tabaci on onion (Table 2). By contrast, on cabbage, no significant differences were found in the durations of any stage or the entire immature stage between the two reproductive modes $(P>0.05$; Table 2$)$.

Survival Rates of Immature Arrhenotokous and Thelytokous T. tabaci on Two Different Host Plants. On onion, the egg hatching rate $(P=0.781)$, the survival rates of the first instar $(P=0.066)$, and pupal stage $(P=0.377)$ were not significantly different between the two reproductive modes (Table 3 ). However, survival rates in the second instar and prepupa stages in thelytokous $T$. tabaci were significantly lower than those in arrhenotokous thrips $(P<0.05$; Table 3). On cabbage, except for a significantly lower egg-hatching rate $(P=0.006)$ in thelytokous T. tabaci, there was no significant difference in sur- vival rates in each stage between the two reproductive modes $(P>0.05$; Table 3$)$.

Fitness and Demographic Parameters of Arrhenotokous and Thelytokous T. tabaci on Two Different Host Plants. No interactions between host plant and the reproductive mode effects were found on female longevity of T. tabaci (Table 4). Host plant significantly affected the longevity of $T$. tabaci while the reproductive mode had no effects (Table 4). By contrast, the total fecundity, daily fecundity, and five demographic parameters had significant host plant-reproductive mode interactions (Table 4).

On onion, there was no difference in female longevity between the two reproductive modes (Table 5). However, arrhenotokous T. tabaci had significantly greater total fecundity and daily fecundity (Table 5). Mean $R_{0}, r_{\mathrm{m}}$, and $\lambda$ were all significantly greater in arrhenotokous $T$. tabaci (Table 5 ). In addition, $T$ and $T_{\mathrm{d}}$ in arrhenotokous $T$. tabaci were significantly lower than those in thelytokous T. tabaci.

By contrast, except for longevity between the two reproductive modes, totally opposite results were found on cabbage. Total fecundity and daily fecundity in thelytokous T. tabaci were much greater on cabbage (Table 5). Mean $R_{0}, r_{\mathrm{m}}$, and $\lambda$ were all significantly greater in thelytokous T. tabaci (Table 5). T and $T_{\mathrm{d}}$ in thelytokous T. tabaci were significantly lower than those in arrhenotokous T. tabaci.

Identification of Arrhenotokous and Thelytokous $\boldsymbol{T}$. tabaci by a Diagnostic PCR Analysis. Sequencing of a 706 bp COI fragment from five arrhenotokous and five

Table 2. The mean developmental times $[\mathrm{d}$, mean $\pm \mathrm{SE}(n)]$ of immature arrhenotokous and thelytokous $T$. tabaci on two different host plants

\begin{tabular}{|c|c|c|c|c|}
\hline \multirow{2}{*}{ Stage } & \multicolumn{2}{|c|}{ Onion } & \multicolumn{2}{|c|}{ Cabbage } \\
\hline & Arrhenotokous & Thelytokous & Arrhenotokous & Thelytokous \\
\hline Egg & $6.6 \pm 0.03(256)$ & $6.7 \pm 0.04(187)$ & $6.5 \pm 0.47(112)$ & $6.5 \pm 0.53(109)$ \\
\hline $\mathrm{Ll}$ & $2.9 \pm 0.04(247)$ & $3.2 \pm 0.05(166)$ & $2.9 \pm 0.45(108)$ & $2.8 \pm 0.40(102)$ \\
\hline L2 & $4.4 \pm 0.09(210)$ & $5.5 \pm 0.15(102)$ & $3.7 \pm 0.10(103)$ & $3.6 \pm 0.06(101)$ \\
\hline $\mathrm{PP}$ & $1.6 \pm 0.03(192)$ & $1.9 \pm 0.05(64)$ & $1.6 \pm 0.02(101)$ & $1.5 \pm 0.02(100)$ \\
\hline $\mathrm{P}$ & $3.6 \pm 0.03(170)$ & $3.8 \pm 0.06(48)$ & $3.4 \pm 0.03(100)$ & $3.5 \pm 0.02(99)$ \\
\hline Egg to adult & $18.9 \pm 0.12(170)$ & $20.7 \pm 0.23(48)$ & $18.0 \pm 0.13(100)$ & $17.8 \pm 0.10(99)$ \\
\hline
\end{tabular}

Nonnormal data. Means at each stage between two host plants in each reproductive mode and between two reproductive modes on each host plant were compared using the nonparametric Mann-Whitney $U$ test at $P<0.05$ level.

$n$, number of replications in each treatment; L1, first instar; L2, second instar; PP, prepupae; P, pupae. 
Table 3. The survival rates $[\%$, mean \pm SE $(n)]$ of immature arrhenotokous and thelytokous $T$. tabaci on two different host plants

\begin{tabular}{|c|c|c|c|c|}
\hline \multirow{2}{*}{ Stage } & \multicolumn{2}{|c|}{ Onion } & \multicolumn{2}{|c|}{ Cabbage } \\
\hline & Arrhenotokous & Thelytokous & Arrhenotokous & Thelytokous \\
\hline Egg & $94.4 \pm 1.5(42)$ & $90.7 \pm 2.8(47)$ & $88.8 \pm 0.5(18)$ & $76.0 \pm 6.1(37)$ \\
\hline $\mathrm{Ll}$ & $96.2 \pm 1.6(39)$ & $84.0 \pm 4.6(42)$ & $97.1 \pm 1.7(18)$ & $92.9 \pm 3.6(33)$ \\
\hline $\mathrm{L} 2$ & $83.6 \pm 3.6(39)$ & $61.6 \pm 5.2(39)$ & $97.6 \pm 1.4(18)$ & $96.0 \pm 1.0(32)$ \\
\hline PP & $91.4 \pm 2.2(38)$ & $57.8 \pm 7.0(35)$ & $96.8 \pm 2.8(18)$ & $96.0 \pm 1.0(32)$ \\
\hline $\mathrm{P}$ & $86.0 \pm 4.5(38)$ & $75.7 \pm 6.9(28)$ & $99.6 \pm 0.4(18)$ & $96.0 \pm 1.0(32)$ \\
\hline
\end{tabular}

Nonnormal data. Means at each stage between two host plants in each reproductive mode and between two reproductive modes on each host plant were compared using the nonparametric Mann-Whitney $U$ test at $P<0.05$ level.

$n$, number of replications in each treatment; L1, first instar; L2, second instar; PP, prepupae; P, pupae.

thelytokous T. tabaci showed one haplotype of the COI fragment in the arrhenotokous colony (GenBank $\mathrm{KJ} 495744$ ) and two haplotypes in the thelytokous colony (GenBank KJ495742 and KJ495743). The COI sequences from the two colonies contained the characteristic reproductive mode-specific SNPs as observed by Kobayashi and Hasegawa (2012). Consequently, our diagnostic PCR analysis using two reproductive mode-specific primers (Kobayashi and Hasegawa 2012) and a general primer accurately differentiated the individuals of the two reproductive modes (Fig. 1).

Competition Experiments in Cages Between Arrhenotokous and Thelytokous T. tabaci. In the control cages of two reproductive modes on two different host plants, no contamination occurred (Fig. 2). In mixed cages, changes in patterns of thelytokous T. tabaci percentages on two host plants over time were significantly different (Fig. 2; $F=109.62$; df $=1 ; P<$ $0.0001)$. Specifically, on onion, the proportion of thelytokous $T$. tabaci decreased over time $(F=363.48$; $\mathrm{df}=1 ; P=0.003)$ and arrhenotokous T. tabaci outcompeted thelytokous thrips by the first sampling date. At the fifth sampling time, only $\approx 10 \%$ of the samples were thelytokous T. tabaci (Fig. 2). By contrast, on cabbage, the proportion of thelytokous $T$. tabaci increased over time $(F=11.50$; df $=1 ; P=$ 0.001 ) and thelytokous T. tabaci were more abundant after the first sampling date. At the fifth sampling time, $>90 \%$ of samples were thelytokous T. tabaci (Fig. 2).

Host Adaption of Arrhenotokous and Thelytokous T. tabaci Between Onion and Cabbage. Arrhenotokous T. tabaci. Except the first instar and prepupa stages ( $P=0.711$ and $P=0.121$, respectively $)$, the durations of egg stage, second instar, and pupae were significantly longer on onion compared with cabbage $(P<$ 0.05 ; Table 2). The entire immature developmental time on onion was $\approx 1 \mathrm{~d}$ longer than that on cabbage (Table 2). There were no significant differences in egg hatching rate $(P=0.922)$ and first instar survival rate $(P=0.774)$ between arrhenotokous $T$. tabaci on onion and cabbage (Table 3). However, the survival rates in the second instar, prepupa, and pupa stages were significantly lower on onion $(P<0.05$; Table 3$)$. Female longevity on cabbage was significantly longer than on onion, but the total fecundity and daily fecundity on onion was significantly greater than on cabbage (Table 5).

There were no differences in mean $R_{0}$ and $T$ between two different host plants (Table 5). However, arrhenotokous $T$. tabaci on onion had a significantly greater $r_{\mathrm{m}}$ and $\lambda$ and lower $T_{\mathrm{d}}$ (Table 5 ).

Thelytokous T. tabaci. The durations of egg, first instar, second instar, prepupa, and pupa stages were significantly longer on onion compared with those on cabbage $(P<0.0001$; Table 2$)$. The entire immature developmental time on onion was $\approx 3 \mathrm{~d}$ longer than that on cabbage (Table 2$)$. The egg-hatching rate $(P<$ $0.0001)$ on onion was higher than that on cabbage (Table 3). Except for the first instar $(P=0.156)$, the survival rates in the second instar, prepupa, and pupa stages were significantly lower on onion $(P<0.0001$; Table 3). Thelytokous T. tabaci females had significantly greater longevity and higher total fecundity and daily fecundity on cabbage (Table 5).

There were significant differences in all five demographic parameters between thelytokous T. tabaci on onion and cabbage (Table 5). Specifically, mean $R_{0}$,

Table 4. Effects of host plant and reproductive mode on fitness and demographic growth parameters of T. tabaci (two-way ANOVA, $P<0.05)$

\begin{tabular}{|c|c|c|c|c|c|c|c|c|}
\hline & Longevity & Total fecundity & Daily fecundity & $R_{0}$ & $T$ & $r_{\mathrm{m}}$ & $\lambda$ & $T_{\mathrm{d}}$ \\
\hline \multicolumn{9}{|l|}{$\mathrm{R}$} \\
\hline F & 0.62 & 0.06 & 0.05 & 0.71 & 0.67 & 0.97 & 0.71 & 10.81 \\
\hline$P$ & 0.433 & 0.815 & 0.825 & 0.400 & 0.414 & 0.326 & 0.399 & 0.001 \\
\hline \multicolumn{9}{|l|}{$\mathrm{H}$} \\
\hline F & 10.42 & 0.06 & 0.48 & 20.65 & 7.31 & 88.60 & 89.26 & 66.55 \\
\hline$P$ & 0.001 & 0.815 & 0.488 & $<0.0001$ & 0.008 & $<0.0001$ & $<0.0001$ & $<0.0001$ \\
\hline \multicolumn{9}{|c|}{$\mathrm{H} \times \mathrm{R}$} \\
\hline$F$ & 0.10 & 8.43 & 17.11 & 43.63 & 14.53 & 165.85 & 167.23 & 116.90 \\
\hline$P$ & 0.759 & 0.004 & $<0.0001$ & $<0.0001$ & $<0.0001$ & $<0.0001$ & $<0.0001$ & $<0.0001$ \\
\hline
\end{tabular}

$\mathrm{H}$, host plant; R, reproductive mode; $R_{0}$, net reproductive rates; $T$, mean generation time; $r_{\mathrm{m}}$, the intrinsic rate of natural increase; $\lambda$, finite rate of increase; $T_{\mathrm{d}}$, population doubling time. 
Table 5. Summary of fitness and demographic growth parameters of arrhenotokous and thelytokous T. tabaci on two different host plants

\begin{tabular}{|c|c|c|c|c|c|c|c|c|c|}
\hline Treatment & $N$ & Longevity & Total fecundity & Daily fecundity & $R_{0}$ & $T$ & $r_{\mathrm{m}}$ & $\lambda$ & $T_{\mathrm{d}}$ \\
\hline A on onion & 69 & $29.8 \pm 1.9 \mathrm{bc}$ & $118.7 \pm 11.0 \mathrm{a}$ & & & & & & \\
\hline $\mathrm{T}$ on onion & 46 & $28.7 \pm 2.4 \mathrm{c}$ & $84.9 \pm 10.0 \mathrm{~b}$ & $2.4 \pm 0.2 \mathrm{~b}$ & $19.8 \pm 2.3 \mathrm{c}$ & $42.4 \pm 1.5 \mathrm{a}$ & $071 \pm 0.002 \mathrm{~d}$ & $1.073 \pm 0.003 \mathrm{~d}$ & $9.8 \pm 0.3 \mathrm{a}$ \\
\hline A on cabbage & 35 & $38.3 \pm 3.0 \mathrm{a}$ & $84.9 \pm 6.1 b$ & $2.3 \pm 0.1 b$ & $45.6 \pm 3.6 \mathrm{~b}$ & $40.3 \pm 1.2 \mathrm{ab}$ & $0.095 \pm 0.002 \mathrm{c}$ & $1.100 \pm 0.003 \mathrm{c}$ & $7.3 \pm 0.2 b$ \\
\hline $\mathrm{T}$ on cabbage & 36 & $35.7 \pm 2.2 \mathrm{ab}$ & $113.6 \pm 8.4 \mathrm{a}$ & $3.2 \pm 0.2 \mathrm{a}$ & $73.2 \pm 5.2 \mathrm{a}$ & $35.0 \pm 0.8 \mathrm{c}$ & $0.123 \pm 0.002 \mathrm{a}$ & $1.131 \pm 0.002 \mathrm{a}$ & $5.6 \pm 0.1 d$ \\
\hline
\end{tabular}

Normal data. Means within the same column followed by the same letters are not significantly different at $P<0.05$ level according to LSD test for multiple comparisons.

A, arrhenotokous T. tabaci; $\mathrm{T}$, thelytokous T. tabaci; $N$, number of replications in each treatment; $R_{0}$, net reproductive rates; $T$, mean generation time; $r_{\mathrm{m}}$, the intrinsic rate of natural increase; $\lambda$, finite rate of increase; $T_{\mathrm{d}}$, population doubling time.

$r_{\mathrm{m}}$, and $\lambda$ were much higher on cabbage, while $T$ and $T_{\mathrm{d}}$ were significantly lower on cabbage (Table 5 )

\section{Discussion}

Co-occurrence of sexual and asexual reproduction is found in various insect species, including parasitoid wasps (Day and Hedlund 1988, Stouthamer et al. 1990, Arakaki and Kinjo 1998, Huigens et al. 2000, Schneider et al. 2002, Copeland et al. 2010), ants (Kellner and Heinze 2011), honey bees (Greeff 1996), aphids (Simon et al. 2002), cockroaches (Corley and Moore 1999), and thrips (Mound 1992, Nakao and Yabu 1998, Nault et al. 2006). Sympatric sexual and asexual populations offer excellent models to evaluate the relative costs and benefits of sex. Theoretically, compared with asexual individuals, sexual ones are less competitive due to the cost of producing males (Maynard Smith 1978). Asexual (e.g., thelytokous) populations have potential advantages, as they do not waste eggs producing males and can establish populations more easily (Stouthamer 1993). However, based on long-term processes, sexual reproduction (e.g., arrhenotoky) has the advantage of preventing deleterious mutation accumulations and increasing environmental adaption (Hurst and Peck 1996).

However, practically, the relative performance of each mode of reproduction varies remarkably and may depend on different factors, including temperature (Wang and Smith 1996), hosts (Day and Hedlund 1988), habitat (Schneider et al. 2002), and infection of

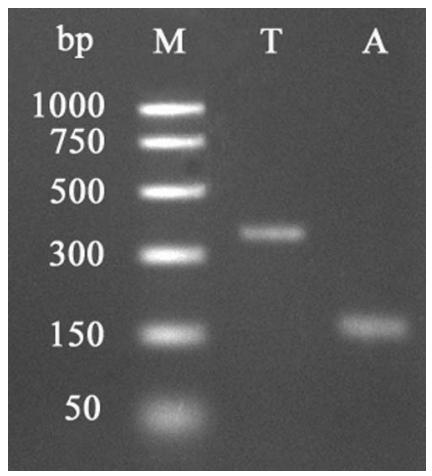

Fig. 1. Reproductive mode-specific PCR products of $T$. tabaci. M, DNA Marker; A, arrhenotokous T. tabaci (161 bp); T, thelytokous T. tabaci (351 bp). endosymbiotic bacteria (Miura and Tagami 2004), and there are no general patterns of increased or decreased fitness parameters in thelytokous populations.

Differences in life table traits between arrhenotokous and thelytokous races have been reported in different insect species. In the parasitoid Trichogramma minutum Riley (Hymenoptera: Trichogrammatidae), thelytokous lines required more time to develop from eggs to adults than arrhenotokous lines. Despite their similar adult life span, thelytokous parasitoids produced significantly fewer offspring than arrhenotokous ones (Wang and Smith 1996). In Trichogramma kaykai Pinto \& Stouthamer reared on Trichoplusia ni (Hübner) (Lepidoptera: Noctuidae) eggs, arrhenotokous wasps produced more progeny and lived longer than their thelytokous counterparts (Hohmann et al. 2001). When reared on Apodemia mormo (C. Felder \& R. Felder) (Lepidoptera: Riodinidae) eggs, thelytokous Tr. kaykai had relatively greater longevity but lower values of $R_{0}$ and $r_{\mathrm{m}}$ (Miura and Tagami 2004). In Thrips nigropilosus Uzel (Thysanoptera: Thripidae), there was no difference in the duration of preovarial maturation, oviposition periods, and egg hatch whereas the arrhenotokous race laid more eggs than the thelytokous race under the same conditions (Nakao and Muraji 2007).

In our study, the performance of arrhenotokous and thelytokous T. tabaci was very different on two host plants. On onion, in thelytokous T. tabaci, the development of the first and second instars, prepupae and pupae was much slower and the mortality of second instars and prepupae was much higher when compared with arrhenotokous thrips. Additionally, thelytokous T. tabaci had significantly lower total and daily fecundity. Lower mean $R_{0}, r_{\mathrm{m}}$, and $\lambda$ and greater $T$ and $T_{\mathrm{d}}$ suggested thelytokous $T$. tabaci is less competitive than its arrhenotokous counterpart on onion. The opposite results were found on cabbage. Although there was no difference in development and survivorship of immature stages between the two reproductive modes (except lower egg hatching rate in thelytokous $T$. tabaci), thelytokous $T$. tabaci performed better on cabbage in total and daily fecundity and five demographic parameters. Additionally, the results of cage experiments were consistent with life table traits. On onion, arrhenotokous T. tabaci outcompeted thelytokous T. tabaci, while on cabbage the opposite occurred. 


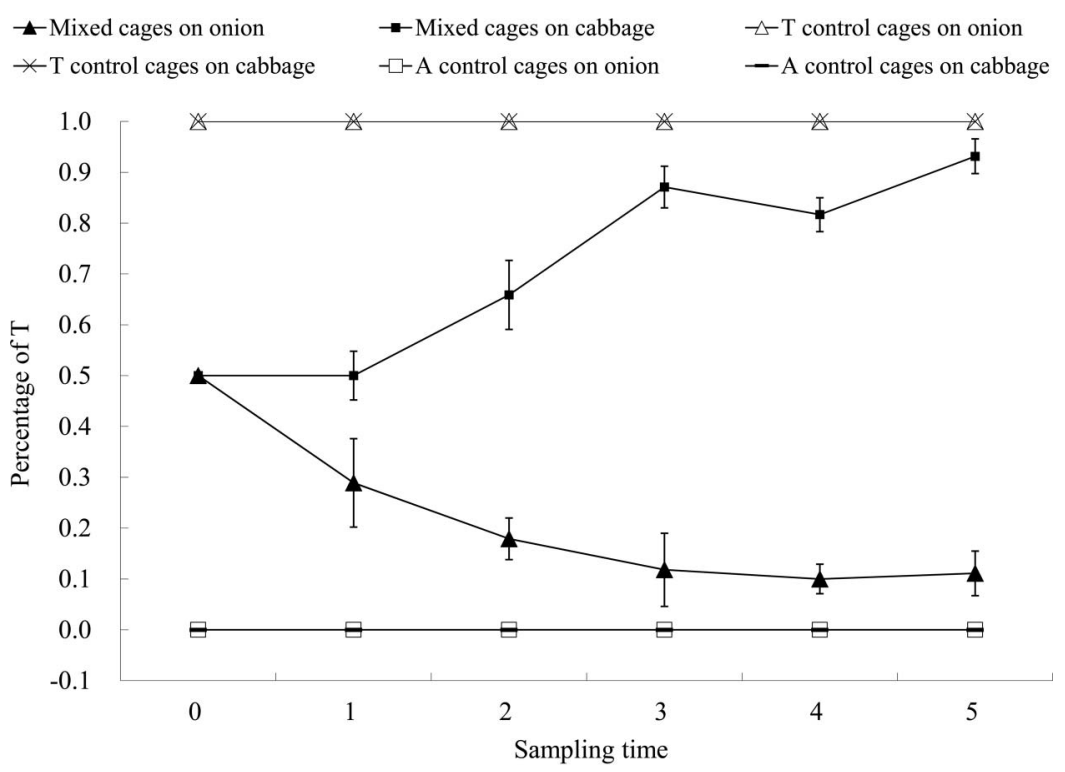

Fig. 2. Changes of thelytokous T. tabaci proportions in mixed populations on onion and cabbage in the laboratory. A arrhenotokous T. tabaci; T, thelytokous T. tabaci.

Different performance of two reproductive modes on different hosts, as noted above for Tr. kaykai on two different Lepidopteran hosts, might be due to a difference in host adaption. Indeed, different host adaption of arrhenotokous and thelytokous populations was reported in other insects (Day and Hedlund 1988). In our case, the results showed that arrhenotokous T. tabaci is better adapted to onion. Although the rates of development in egg, second instar, and pupa stages; survivorship in second instar, prepupa, and pupa stages; and female adult longevity were significantly lower on onion than on cabbage, greater fecundity on onion still allowed the arrhenotokous $T$. tabaci on onion to have a significantly greater $r_{\mathrm{m}}, \lambda$, and lower $T_{\mathrm{d}}$. By contrast, thelytokous T. tabaci is better adapted to cabbage. Except for a lower egghatching rate and the similar first instar survival rate, thelytokous T. tabaci had significantly shorter developmental times and dramatically greater survival rates in all immature stages on cabbage. Furthermore, thelytokous T. tabaci female adults lived significantly longer and laid more eggs on cabbage. Comparison of five demographic parameters between onion and cabbage suggested thelytokous T. tabaci is more adapted to cabbage than to onion.

It is worth noting that there were no significant differences in longevity between the two reproductive modes both on onion and cabbage. However, both arrhenotokous and thelytokous T. tabaci females had shorter longevity on onion. Additionally, T. tabaci from both reproductive modes had lower survival rates during immature stages on onion than on cabbage. This suggests that onion had some negative effects on the development of both arrhenotokous and thelytokous T. tabaci. Thelytokous T. tabaci was more sensitive to onion and the mortality was $\approx 60 \%$ during second instar and prepupa stages.
Host-related performance differences and distinctive genetic differences between arrhenotokous and thelytokous $T$. tabaci suggest that these two reproductive modes have different evolutionary histories. Based on host-plant adaption and genetic differentiation, T. tabaci was divided into three major evolutionary lineages; two were clearly associated with leek and the third with tobacco and they had an ancient origin and long-term isolation (Brunner et al. 2004). An analysis of the rate of evolution suggested that an ancient arrhenotokous strain differentiated into two types, the tobacco and leek types, and then the leek type diverged into an arrhenotokous type and a thelytokous type (Brunner et al. 2004, Toda and Murai 2007). The two populations we used in this study belong to the latter two lineages, arrhenotokous leek type and thelytokous leek type. A previous study suggested that the tobacco type and leek types had strong host-specific adaptations to tobacco and leek (Chatzivassiliou et al. 2002). In our study, two leek types, arrheotokous and thelytokous T. tabaci, showed differences in host adaption to onion and cabbage. The phylogenetic and evolution rate analysis suggested the tobacco type diverged earlier (around 28 million years ago) from the leek type (Brunner et al. 2004). This divergence is likely due to different host adaption. Around 7 million years later, the leek type diverged into two types (Brunner et al. 2004). This subsequent diversification might also be associated with host adaption. Better performance of arrhenotokous T. tabaci on onion and better performance of thelytokous $T$. tabaci on cabbage provide evidence for this hypothesis. However, the taxa of these three lineages are still not clear. Genetic variability and pairwise $F_{S T}$ statistics suggested these three $T$. tabaci lineages might be considered different cryptic (sub) species (Brunner et al. 2004), and phylogeny based on nuclear genes confirms 
that the sexual and asexual leek lineages, i.e., the arrhenotokous and thelytokous lineages, are genetically isolated (Kobayashi et al. 2013). However, evidence only based on pairwise genetic differences (i.e., $F_{S T}$ ) is not sufficient for the estimation of actual gene flow or hybridization (Dres and Mallet 2002) and additional gene flow studies are required to clarify relationships of these sympatric taxa.

Differences in performance by arrhenotokous and thelytokous T. tabaci on onion and cabbage provide useful knowledge about the roles of two reproductive modes in the population dynamics on different crops. Based on the crops and T. tabaci population structure in the field, it should be possible to use this knowledge to predict their potential population dynamics and associated crop damage. For example, studies have reported the increasing incidence of insecticide resistance in T. tabaci (Diaz-Montano et al. 2011). Difference in reproduction may affect insecticide-resistant alleles in the population, and hence the ability to control T. tabaci populations using specific insecticides. Additionally, the increasing incidence of virus diseases transmitted by T. tabaci has been documented (Diaz-Montano et al. 2011). Arrhenotokous and thelytokous populations have been shown to differ in their ability to transmit some of these plant viruses (Chatzivassiliou et al. 1999, 2002; Tedeschi et al. 2001; Westmore et al. 2013). Consequently, the population structure of $T$. tabaci on different crops can affect the overall risk of crop damage as well as affect the tactics used to mitigate such damage.

In conclusion, the performance of arrhentokous and thelytokous T. tabaci was host-plant dependent. Results from life table and cage experiments showed that on onion, arrhenotokous T. tabaci performed better than thelytokous T. tabaci, while on cabbage the opposite occurred. When comparing life table and demographic growth parameters on different host plants, we found that arrhenotokous T. tabaci was better adapted to onion, whereas thelytokous T. tabaci was better adapted to cabbage. Our results provide important knowledge about the relationships and evolution of these two reproductive modes and their population dynamics on different crops. We suggest that this knowledge will also be useful for pest management strategies.

\section{Acknowledgments}

We thank Beth Cole, Hobart and William Smith Colleges, for her assistance conducting this study, and H. L. Collins (Cornell University) for help in editing the manuscript. This project was supported by the China Scholarship Council (CSC) and New York State Cabbage Research Association.

\section{References Cited}

Arakaki, N., and K. Kinjo. 1998. Notes on the parasitoid fauna of the serpentine leafminer Liriomyza trifolii (Burgess) (Diptera: Agromyzidae) in Okinawa, southern Japan. Appl. Entomol. Zool. 33: 577-581.

Brunner, P. C., E. K. Chatzivassiliou, N. I. Katis, and J. E. Frey. 2004. Host-associated genetic differentiation in
Thrips tabaci (Insecta; Thysanoptera), as determined from mtDNA sequence data. Heredity 93: 364-370.

Carey, J. R. 1993. Applied demography for biologists with special emphasis on insects. Oxford University Press, New York, NY

Chatzivassiliou, E. K., T. Nagata, N. I. Katis, and D. Peters. 1999. Transmission of tomato spotted wilt tospovirus by Thrips tabaci populations originating from leek. Plant Pathol. 48: 700-706.

Chatzivassiliou, E. K., D. Peters, and N. I. Katis. 2002. The efficiency by which Thrips tabaci populations transmit Tomato spotted wilt virus depends on their host preference and reproductive strategy. Phytopathology 92: 603609.

Copeland, C. S., M. A. Hoy, A. Jeyaprakash, M. Aluja, R. Ramirez-Romero, and J. M. Sivinski. 2010. Genetic characteristics of bisexual and female-only populations of Odontosema anastrephae (Hymenoptera: Figitidae). Fla. Entomol. 93: 437-443.

Corley, L. S., and A. J. Moore. 1999. Fitness of alternative modes of reproduction: developmental constraints and the evolutionary maintenance of sex. Proc. R. Soc. Biol. Sci. Ser. B 266: 471-476.

Day, W. H., and R. C. Hedlund. 1988. Biological comparisons between arrhenotokous and thelytokous biotypes of Mesochorus nigripes [Hym.: Ichneumonidae]. Entomophaga 33: 201-210.

Diaz-Montano, J., M. Fuchs, B. A. Nault, J. Fail, and A. M. Shelton. 2011. Onion thrips (Thysanoptera: Thripidae): a global pest of increasing concern in onion. J. Econ. Entomol. 104: 1-13.

Dorit, R. L., O. Ohara, C. B.-C. Hwang, J. B. Kim, and S. Blackshaw. 2001. Direct DNA Sequencing of PCR Products. Curr. Protoc. Mol. Biol. 15.2: 1-15.

Dres, M., and J. Mallet. 2002. Host races in plant-feeding insects and their importance in sympatric speciation. Philos. Trans. R. Soc. Lond. B. Biol. Sci. 357: 471-492.

Fekrat, L., P. Shishehbor, S. Manzari, and E. S. Nejadian. 2009. Comparative development, reproduction and life table parameters of three populations of Thrips tabaci (Thysanoptera: Thripidae) on onion and tobacco. J. Entomol. Soc. Iran 29: 11-23.

Greeff, J. M. 1996. Thelytokous versus arrhenotokous worker reproduction in the cape honeybee and other eusocial Hymenoptera. Hereditas 124: 99-103.

Hebert, P.D.N., E. H. Penton, J. M. Burns, D. H. Janzen, and W. Hallwachs. 2004. Ten species in one: DNA barcoding reveals cryptic species in the neotropical skipper butterfly Astraptes fulgerator. Proc. Natl. Acad. Sci. U.S.A. 101: 14812-14817.

Hohmann, C. L., R. F. Luck, and R. Stouthamer. 2001. Host deprivation effect on reproduction and survival of Wolbachia-infected and uninfected Trichogramma kaykai Pinto \& Stouthamer (Hymenoptera: Trichogrammatidae). Neotrop. Entomol. 30: 601-605.

Huigens, M. E., R. F. Luck, R.H.G. Klaassen, M. Maas, M. Timmermans, and R. Stouthamer. 2000. Infectious parthenogenesis. Nature 405: 178-179.

Hurst, L. D., and J. R. Peck. 1996. Recent advances in understanding of the evolution and maintenance of sex. Trends Ecol. Evol. 11: A46-A52.

Jacobson, A. L., and G. G. Kennedy. 2013. Specific insectvirus interactions are responsible for variation in competency of different thrips tabaci isolines to transmit different tomato spotted wilt virus isolates. PLoS ONE 8: e54567.

Jenser, G., and Á. Szénási. 2004. Review of the biology and vector capability of Thrips tabaci Lindeman (Thys- 
anoptera: Thripidae). Acta Phytopathol. Entomol. Hung. 39: $137-155$.

Kellner, K., and J. Heinze. 2011. Mechanism of facultative parthenogenesis in the ant Platythyrea punctata. Evol. Ecol. 25: 77-89.

Kobayashi, K., and E. Hasegawa. 2012. Discrimination of reproductive forms of Thrips tabaci (Thysanoptera: Thripidae) by PCR with sequence specific primers. J. Econ. Entomol. 105: 555-559.

Kobayashi, K., J. Yoshimura, and E. Hasegawa. 2013. Coexistence of sexual individuals and genetically isolated asexual counterparts in a thrips. Sci. Rep. 3: 3286.

Maia, A.D.N., A.J.B. Luiz, and C. Campanhola. 2000. Statistical inference on associated fertility life table parameters using jackknife technique: Computational aspects. J. Econ. Entomol. 93: 511-518.

Maynard Smith, J. 1978. The evolution of sex. Cambridge University Press, Cambridge, MA.

Meyer, J. S., C. G. Ingersoll, L. L. McDonald, and M. S. Boyce. 1986. Estimating uncertainty in population growth rates: jackknife vs. bootstrap techniques. Ecology 67: 11561166.

Miura, K., and Y. Tagami. 2004. Comparison of life history characters of arrhenotokous and Wolbachia-associated thelytokous Trichogramma kaykai Pinto and Stouthamer (Hymenoptera: Trichogrammatidae). Ann. Entomol. Soc. Am. 97: 765-769.

Moritz, G. 1997. Structure, growth and development, pp. 15-64. In T. Lewis (ed.), Thrips as Crop Pests. CAB International, New York, NY.

Mound, L. A. 1992. Patterns of sexuality in Thysanoptera. pp. 2-14. In E. A. Cameron, D. A. Teulon, L. H. McCormick and T. E. Kolb (eds.), The 1991 Conference on Thrips (Thysanoptera): Insect and Disease Considerations in Sugar Maple Management. U.S. Dep. Agric. Forest Service, General Technical Report, University Park, PA.

Nakao, S., and S. Yabu. 1998. Ethological and chemical discrimination between thelytokous and arrhenotokous Thrips nigropilosus Uzel, with discussion of taxonomy. Jpn. J. Appl. Entomol. Zool. 42: 77-83.

Nakao, S., and M. Muraji. 2007. Life history traits and nucleotide sequences of mt-DNA of thelytokous and arrhenotokous races of Thrips nigropilosus uzel. J. Insect Sci. 7: 27-28.

Nault, B. A., A. M. Shelton, J. L. Gangloff-kaufmann, M. E. Clark, J. L. Werren, J. C. Cabrera-la Rosa, and G. G. Kennedy. 2006. Reproductive modes in onion thrips (Thysanoptera: Thripidae) populations from New York onion fields. Environ. Entomol. 35: 1264-1271.

Sakimura, K. 1962. The present status of thrips-borne viruses, pp. 33-40. In K. Maramorosch (ed.), Biological transmission of disease agents. Academic, New York, NY.

Schneider, M. V., L. W. Beukeboom, G. Driessen, L. Lapchin, C. Bernstein, and J.J.M. Van Alphen. 2002. Geographical distribution and genetic relatedness of sympatrical thelytokous and arrhenotokous populations of the parasitoid Venturia canescens (Hymenoptera). J. Evol. Biol. 15: 191-200.
Shelton, A. M., W. T. Wilsey, and M. A. Schmaedick. 1998. Management of onion thrips (Thysanoptera: Thripidae) on cabbage by using plant resistance and insecticides. J. Econ. Entomol. 91: 329-333.

Simon, J. C., C. Rispe, and P. Sunnucks. 2002. Ecology and evolution of sex in aphids. Trends Ecol. Evol. 17: 34-39.

SPSS Inc. 2007. SPSS for Windows, Version 16.0. SPSS Inc. Chicago, IL.

Stouthamer, R. 1993. The use of sexual versus asexual wasps in biological control. Entomophaga 38: 3-6.

Stouthamer, R., J. D. Pinto, G. R. Platner, and R. F. Luck. 1990. Taxonomic status of thelytokous forms of Trichogramma (Hymenoptera: Trichogrammatidae). Ann. Entomol. Soc. Am. 83: 475-481.

Tedeschi, R., M. Ciuffo, G. Mason, P. Roggero, and L. Tavella. 2001. Transmissibility of four tospoviruses by a thelytokous population of Thrips tabaci from Liguria, Northwestern Italy. Phytoparasitica 29: 37-45.

Tiewsiri, K., and P. Wang. 2011. Differential alteration of two aminopeptidases $\mathrm{N}$ associated with resistance to $\mathrm{Ba}$ cillus thuringiensis toxin Cry1Ac in cabbage looper. Proc. Natl. Acad. Sci. U.S.A. 108: 14037-14042.

Toda, S., and T. Murai. 2007. Phylogenetic analysis based on mitochondrial COI gene sequences in Thrips tabaci Lindeman (Thysanoptera: Thripidae) in relation to reproductive forms and geographic distribution. Appl. Entomol. Zool. 42: 309-316.

Trdan, S., N. Valič, and D. Žnidarčič. 2007. Field efficacy of deltamethrin in reducing damage caused by Thrips tabaci Lindeman (Thysanoptera: Thripidae) on early white cabbage. J. Pest Sci. 80: 217-223.

Trdan, S., D. Znidarcic, M. Kac, and M. Vidrih. 2008. Yield of early white cabbage grown under mulch and nonmulch conditions with low populations of onion thrips (Thrips tabaci Lindeman). Int. J. Pest Manage. 54: 309 318.

Wang, Z. Y., and S. M. Smith. 1996. Phenotypic differences between thelytokous and arrhenotokous Trichogramma minutum from Zeiraphera canadensis. Entomol. Exp. Appl. 78: 315-323.

Westmore, G. C., F. S. Poke, G. R. Allen, and C. R. Wilson. 2013. Genetic and host-associated differentiation within Thrips tabaci Lindeman (Thysanoptera: Thripidae) and its links to Tomato spotted wilt virus-vector competence. Heredity 111: 210-215.

Wijkamp, I., N. Almarza, R. Goldbach, and D. Peters. 1995. Distinct levels of specificity in thrips transmission of tospoviruses. Phytopathology 85: 1069-1074.

Wolfenbarger, D., and E. T. Hibbs. 1958. Onion thrips (Thrips tabaci Lind.) infesting cabbage. J. Econ. Entomol. 51: 394-396.

Zawirska, I. 1976. Untersuchungen über zwei biologische Typen von Thrips tabaci Lind. (Thysanoptera, Thripidae) in der VR Polen. Arch. Phytopathol. Plant Prot. 12: 411422.

Received 27 February 2014; accepted 19 May 2014. 